\title{
Planned Concomitant Left and Right Ventricular Assist Device Insertion to Avoid Long-term Biventricular Mechanical Support: Bridge to Right Ventricular Recovery
}

\author{
Michael Salna, MD,$^{1^{*}}$ Yasuhiro Shudo, $\mathrm{MD}, \mathrm{PhD},{ }^{1^{*}}$ Jeffrey J. Teuteberg, $\mathrm{MD},{ }^{2}$ Dipanjan Banerjee, $\mathrm{MD},{ }^{2}$ \\ Richard V. Ha, MD, ${ }^{1}$ Y. Joseph Woo, MD, ${ }^{1}$ William Hiesinger, MD ${ }^{1}$ \\ ${ }^{1}$ Department of Cardiothoracic Surgery, Stanford University School of Medicine, Stanford, CA; ${ }^{2}$ Department of Medicine, \\ Division of Cardiovascular Medicine, Stanford University School of Medicine, Stanford, CA
}

\section{ABSTRACT}

Introduction: The planned use of a temporary right ventricular assist device (RVAD) at the time of left ventricular assist device (LVAD) implantation may prevent the need for a permanent biventricular assist device (BiVAD). Herein we describe our RVAD weaning protocol that was effectively employed in 4 patients to prevent the need for permanent BiVAD.

Methods: Four patients in refractory cardiogenic shock underwent planned RVAD insertion during LVAD implantation due to severely depressed right ventricular function with dilation preoperatively. A standardized RVAD weaning protocol was employed in these 4 patients in preparation for decannulation.

Results: Temporary RVADs were successfully placed in all 4 patients at the time of LVAD implantation. All patients survived to RVAD decannulation and discharge and were alive at the time of most recent follow-up (range, 528-742 days post-RVAD decannulation).

Conclusion: Planned implantation of a temporary RVAD in high risk patients may avoid the need for biventricular mechanical support in the future

\section{INTRODUCTION}

Heart failure is a leading cause of death in the United States with an estimated $50 \%$ mortality within 5 years of diagnosis [Yancy 2013]. In advanced end-stage heart failure refractory to medical therapy, the use of a left ventricular assist device (LVAD) has become an established standard of care [Rose 2001].

Despite the success of LVAD therapy, nearly one third of patients develop right ventricular (RV) dysfunction/ failure after device implantation [Kirklin 2013]. RV failure continues to be a significant cause of perioperative morbidity and mortality and oftentimes necessitates the

Received May 3, 2018; received in revised form August 6, 2018; accepted August 9, 2018.

*Both authors contributed equally to this work.

Correspondence: William Hiesinger, MD, Department of Cardiothoracic Surgery, Stanford University School of Medicine, 300 Pasteur Drive, Stanford, CA 94305; 1-650-725-4497; fax: 1-650-736-0901 (e-mail: willhies@stanford.edu). insertion of a right ventricular assist device (RVAD) to support systemic perfusion.

Although many forms of RV failure are reversible, the unplanned implantation of an RVAD portends very poor outcomes [Kirklin 2013; Takeda 2014]. In this report, we describe the planned concomitant insertion of an RVAD during LVAD implantation in 4 patients thought to have high risk of developing postprocedural RV failure and describe our algorithm in weaning patients from this temporary support once the right ventricle has recovered. This strategy may be an effective means of avoiding the use of biventricular assist devices (BiVAD), which have been associated with a nearly 70\% 6-month morality rate [Levin 2016].

\section{MATERIALS AND METHODS}

There were 4 patients in refractory cardiogenic shock with severe multiorgan system failure who underwent planned RVAD insertion, with right-atrial to direct main-PA cannulation, during LVAD implantation (Table). Cannulas were secured to their respective sites with 2 circumferentially placed, pledgeted 4-0 polypropylene purse-string sutures and reinforced with tightened tourniquets. To permit ambulation, the PA cannula was tunneled through the third intercostal space, and the venous cannula through the subxiphoid space, thereby also enabling primary sternotomy closure. We do not routinely employ any special techniques or use prosthetic material, such as Gore-Tex, at the time of implantation to facilitate future planned resternotomy. All 4 patients were on preoperative inotropes and had moderate to severely depressed RV function with RV dilation on preoperative echocardiography. After LVAD/RVAD implantation, all patients were ambulatory and able to be successfully weaned from RVAD support by using our weaning protocol outlined below. Preoperative prediction of RV function after LVAD implantation was crucial for device selection and patient outcome. Primary considerations were RV systolic function, degree of dilation, and the pulmonary artery pulsatility index (PAPi). Our team has demonstrated the utility of preoperative PAPi as a novel hemodynamic index for predicting RV failure after LVAD implantation [Kang 2016]. Of the 83 patients in that study, none with PAPi scores $>3.1$ required an RVAD after LVAD implantation, and 74\% of those with PAPi scores $<2.0$ eventually required RVAD spport. In our 
Patients Undergoing Temporary RVAD Implantation at the Time of LVAD Implantation*

\begin{tabular}{|c|c|c|c|c|c|c|c|c|c|c|c|c|c|c|}
\hline 54 & Male & $\begin{array}{l}\text { Ischemic } \\
\text { cardiomy- } \\
\text { opathy }\end{array}$ & $\begin{array}{l}\text { Bridge } \\
\text { to can- } \\
\text { didacy }\end{array}$ & $\begin{array}{c}\text { Moder- } \\
\text { ate }\end{array}$ & $\begin{array}{l}\text { Moder- } \\
\text { ate-se- } \\
\text { vere }\end{array}$ & $\dagger$ & $\dagger$ & 15 & $\dagger$ & $\begin{array}{l}\text { LVAD } \\
\text { and } \\
\text { RVAD }\end{array}$ & $\begin{array}{l}\text { Heart- } \\
\text { Ware }\end{array}$ & $\begin{array}{c}\text { Tandem- } \\
\text { Heart }\end{array}$ & 33 & $\begin{array}{l}\text { LVAD } \\
\text { support } \\
\text { only }\end{array}$ \\
\hline 37 & Male & $\begin{array}{l}\text { Familial } \\
\text { cardiomy- } \\
\text { opathy }\end{array}$ & $\begin{array}{l}\text { Bridge } \\
\text { to can- } \\
\text { didacy }\end{array}$ & Severe & Severe & 51 & 31 & 25 & 0.8 & $\begin{array}{l}\text { LVAD } \\
\text { and } \\
\text { RVAD }\end{array}$ & $\begin{array}{l}\text { Heart- } \\
\text { Mate II }\end{array}$ & None & 30 & $\begin{array}{l}\text { LVAD } \\
\text { support } \\
\text { only }\end{array}$ \\
\hline 30 & Male & $\begin{array}{l}\text { Non- } \\
\text { ischemic } \\
\text { cardiomy- } \\
\text { opathy }\end{array}$ & $\begin{array}{l}\text { Desti- } \\
\text { nation } \\
\text { therapy }\end{array}$ & Severe & Severe & 31 & 21 & 20 & 0.5 & $\begin{array}{l}\text { LVAD } \\
\text { and } \\
\text { RVAD }\end{array}$ & $\begin{array}{l}\text { Heart- } \\
\text { Ware }\end{array}$ & None & 18 & $\begin{array}{l}\text { LVAD } \\
\text { support } \\
\text { only }\end{array}$ \\
\hline
\end{tabular}

*RVAD, right ventricular assist device; LVAD, left ventricular assist device; PAP, pulmonary arterial pressure; CVP, central venous pressure; PAPi, pulmonary artery pulsatility index; VA ECMO, venoarterial extracorporeal membrane oxygenation.

†Data not available to calculate.

current series, the preoperative PAPi scores were 0.8, 0.5, 0.4, and one which was unable to be calculated (patient had severe mitral regurgitation, was on a TandemHeart device, and failed ProtekDuo support). All of these patients were on preoperative inotropic medications from which the patients were gradually weaned, if possible, after implantation.

Our RVAD weaning protocol is as follows: RVAD flow is set to $3.0-3.5$ liters per $\mathrm{min} / \mathrm{m}^{2}$, and moderate inotropic support with epinephrine $(\leq 0.05 \mu \mathrm{g} / \mathrm{kg}$ per min), dopamine $(\leq 5 \mu \mathrm{g} / \mathrm{kg}$ per min), or milrinone ( $\leq 0.5 \mu \mathrm{g} / \mathrm{kg}$ per min) is initiated in the ICU before weaning. RVAD flow was decreased by $0.5 \mathrm{~L} / \mathrm{min}$ every 8 hours until reaching $2.0 \mathrm{~L} / \mathrm{min}$. Simultaneously, LVAD speeds are adjusted to maintain a cardiac index of $2.4 \mathrm{~L}$ per $\mathrm{min} / \mathrm{cm}^{2}$. If patients tolerate $2.0 \mathrm{~L} / \mathrm{min}$ in the ICU, they are brought to the operating room. Under general anesthesia and transesophageal echocardiographic guidance, a redo sternotomy is performed, and RVAD flow is decreased by $0.5 \mathrm{~L} / \mathrm{min}$ every 15 minutes to $0.5 \mathrm{~L} / \mathrm{min}$ of flow with adequate anticoagulation. Pulmonary vasodilators, namely epoprostenol, are regularly used to ensure sufficient RV afterload reduction. Weaning was considered successful if the central venous pressure (CVP) remained at $\leq 15 \mathrm{mmHg}$ with stable LVAD flow. The cannulas of the RVAD are clamped and flushed with heparinized saline. If hemodynamics and RV function via echocardiography are adequate, the RVAD cannulas are removed.

\section{RESULTS}

All 4 patients were discharged to home, and none required BIVAD support by the date of the most recent follow-up (range, 528-742 days post-RVAD decannulation). Duration of RVAD support for the 4 patients (nos. 1, 2, 3, and 4) was 33 days, 30 days, 18 days, and 29 days, respectively. Patients nos. 1 and 3 eventually underwent heart transplantation, and nos. 2 and 4 were stable on isolated LVAD support. Patient no. 4 required readmission for inpatient diuretic therapy secondary to RV failure after an episode of VT arrest 4 months after discharge. He remained on outpatient IV dobutamine and an aggressive oral diuretic regimen for a year after discharge.

\section{DISCUSSION}

The goal of early and planned RVAD insertion at the time of LVAD implantation is to temporarily support a failing RV until it has sufficiently recovered. Ultimately, this may reduce the need for a long-term BiVAD in the future. We have implemented this strategy-both in early implantation and use of a standardized weaning protocol-with 4 patients to date, and it has yielded good results.

Right ventricular failure after LVAD implantation continues to be a major complication associated with poor outcomes. Mortality rates are particularly high owing to the critical condition of these patients stemming from preexisting complications such as bleeding, coagulopathy, pulmonary hypertension, liver failure, renal failure, and other end-organ damage. Therefore, it is essential to not delay RVAD initiation for risk of further end-organ damage from persistent worsening malperfusion [Takeda 2014]. Thus, we believe that prophylactic planned temporary RVAD insertion at the time of LVAD implantation is favorable in patients deemed to be at 
high risk for RV failure as determined by factors such as preoperative RV systolic function, dilation, and PAPi. Moreover, the RVAD circuit permits the integration of an oxygenator in cases of pulmonary failure.

There are several limitations to this strategy including greater operative risk and morbidity and the need for redo sternotomy at the time of decannulation. Two recently introduced percutaneous options-the Impella RP® (Abiomed) and ProtekDuo® (TandemLife) cannula-may help to negate these risks and facilitate earlier, more aggressive RV support. The Impella RP is a microaxial pump, inserted through the femoral vein and traversing the tricuspid and pulmonic valves, which receives inflow from the inferior vena cava and provides outflow of up to $4 \mathrm{~L} / \mathrm{min}$ through the pulmonary artery. It has been shown to provide reliable and sustained hemodynamic support with improvements in RA pressure, cardiac index, and the need for inotropic support in right heart failure [Anderson 2015]. The ProtekDuo is a flexible, duallumen transjugular cannula that can be combined with the TandemHeart pump and provides right atrial venous drainage through an outer lumen (29 Fr) and outflow through a cannula $(16 \mathrm{Fr})$ into the pulmonary artery. This not only provides up to $4.5 \mathrm{~L} / \mathrm{min}$ of flow but also facilitates ambulation and easier removal because of its single jugular venous cannulation site [Ravichandran 2018].

In conclusion, we believe that the planned use of a temporary RVAD at the time of LVAD implantation, coupled with an effective standardized weaning protocol, can permit RV recovery and subsequently prevent the need for a BiVAD in the future. Although this study is limited by its small sample size, we believe that more robust investigations into the use of prophylactic RVADs may improve outcomes in this critically ill population and further validate predictors of RV failure after LVAD implantation.

\section{REFERENCES}

Anderson B, Goldstein J, Milano C, et al. 2015. Benefits of a novel percutaneous ventricular assist device for right heart failure: The prospective RECOVER RIGHT study of the Impella RP device. J Heart Lung Transplant 34:1549-60

Kang G, Ha R, Banerjee D. 2016. Pulmonary artery pulsatility index predicts right ventricular failure after left ventricular assist device implantation. J Heart Lung Transplant 35:67-73.

Kirklin JK, Naftel DC, Kormos RL, et al. 2013. Fifth INTERMACS annual report: risk factor analysis from more than 6,000 mechanical circulatory support patients. J Heart Lung Transplant 32:141-56.

Levin AP, Jaramillo N, Garan AR, et al. 2016. Outcomes of contemporary mechanical circulatory support device configurations in patients with severe biventricular failure. J Thorac Cardiovasc Surg 151:530-535.e2.

Ravichandran AK, Baran DA, Stelling K, Cowger JA, Salerno CT. 2018. Outcomes with the Tandem Protek Duo dual-lumen percutaneous right ventricular assist device. ASAIO J 64:570-2.

Rose EA, Gelijns AC, Moskowitz AJ, et al. 2001. Long-term use of a left ventricular assist device for end-stage heart failure. N Engl J Med 345:1435-43.

Takeda K, Naka Y, Yang JA, et al. 2014. Outcome of unplanned right ventricular assist device support for severe right heart failure after implantable left ventricular assist device insertion. J Heart Lung Transplant $33: 141-8$.

Yancy CW, Jessup M, Bozkurt B, et al. 2013.2013 ACCF/AHA guideline for the management of heart failure: a report of the American College of Cardiology Foundation/American Heart Association Task Force on Practice Guidelines. J Am Coll Cardiol 62:e147-239. 